\title{
On the Equatorial Mass-Loss Rate of Be Stars
}

\section{A.T. Okazaki}

Faculty of Engineering, Hokkai-Gakuen University, Toyohira-ku, Sapporo 062-8605, Japan

\begin{abstract}
We numerically study the formation process of equatorial disks around isolated Be stars, using an SPH code. We find that the viscous decretion disk model naturally explains the observed disk formation episode of $\mathrm{X}$ Per, giving the equatorial mass-loss rate of several $\times 10^{-9}$ $\left(\rho_{0} / 10^{-10} \mathrm{~g} \mathrm{~cm}^{-3}\right) M_{\odot} \mathrm{yr}^{-1}$, where $\rho_{0}$ is the base density of the disk.
\end{abstract}

\section{Introduction}

The circumstellar envelope of Be stars has two components: a polar wind and an equatorial disk. The former is a low-density, fast outflow driven by strong lines (e.g., Castor et al., 1975), whereas the latter is a high-density plasma rotating at a near-Keplerian speed. The mass-loss rate in the equatorial disk has been measured only during the disk formation phase. Telting et al. (1998) found that for X Per, the equatorial mass-loss rate was as high as $4-5 \times 10^{-9} M_{\odot} \mathrm{yr}^{-1}$ for $2 \mathrm{yr}$. The purpose of this paper is to examine numerically the equatorial massloss rate during the disk formation phase and compare it with the observed value, based on the viscous decretion disk model (Lee et al., 1991), which is consistent with many observed features.

\section{Numerical method and assumptions}

The simulations presented here were performed with a 3D SPH code. The artificial viscosity was adjusted so as to keep the Shakura-Sunyaev viscosity parameter $\alpha$ constant. To model the mass ejection from the Be star, particles rotating at the Keplerian velocity were injected, at a constant rate, at $r=$ $1.02 R_{*}$, where $R_{*}$ is the stellar radius. We took a star of $M_{*}=18 M_{\odot}, R_{*}=$ $8 R_{\odot}$, and $T_{\text {eff }}=26000 \mathrm{~K}$. The Be disk was assumed to be isothermal at $1 / 2 T_{\text {eff }}$.

\section{Disk structure}

Fig. 1 shows the surface density evolution for $\alpha=0.1$ (left) and $\alpha=1$ (right). We note that the disk structure gradually approaches that of steady state $\left(\Sigma \propto r^{-2}\right)$. As expected, the radial velocity is subsonic and the disk is near-Keplerian. 

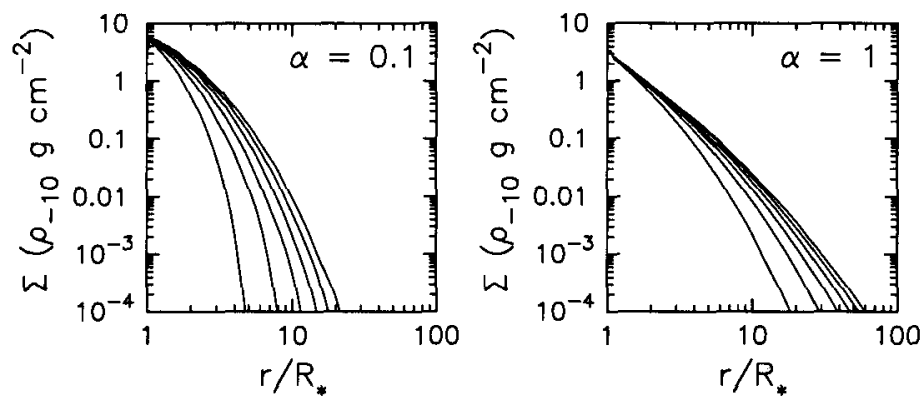

Figure 1. Evolution of the surface-density distribution for $\alpha=0.1$ (left) and $\alpha=1$ (right). The time interval between adjacent contours is $0.5 \mathrm{yr}(t=0.5 \mathrm{yr}, 1 \mathrm{yr}, \ldots, 3 \mathrm{yr}$ from left to right $)$.
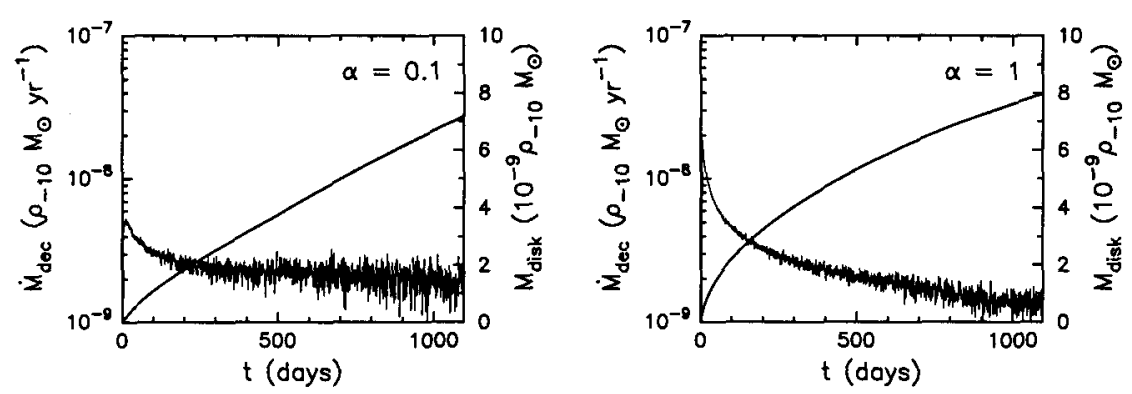

Figure 2. Evolution of the decretion rate $\dot{M}_{\text {dec }}$ (thin line) and the disk mass $M_{\text {disk }}$ (thick line) for $\alpha=0.1$ (left) and $\alpha=1$ (right). The decretion rate is measured at $r=1.04 R_{*}$ and averaged over $\sim 9$ days.

\section{Decretion rate}

Figure 2 shows the change in the decretion rate $\dot{M}_{\text {dec }}$ and the disk mass $M_{\text {disk }}$ during the disk formation phase for $\alpha=0.1$ (left) and $\alpha=1$ (right). As the disk grows, the decretion rate decreases. For the first several years, $\dot{M}_{\text {dec }} \sim$ several $\times 10^{-9}\left(\rho_{0} / 10^{-10} \mathrm{~g} \mathrm{~cm}^{-3}\right) M_{\odot} \mathrm{yr}^{-1}$. Note that the relation between $\dot{M}_{\mathrm{dec}}$ and $\rho_{0}$ is consistent with that observed for X Per.

\section{References}

Castor, J.I., Abbott, D.C., \& Klein, R.I. 1975, ApJ, 195, 157

Lee, U., Osaki, Y., \& Saio, H. 1991, MNRAS, 250, 432

Telting, J.H., Waters, L.B.F.M., Roche, P., Boogert, A.C.A., Clark, J.S., de Martino, D., \& Persi P. 1998, A\&A, 296, 785 